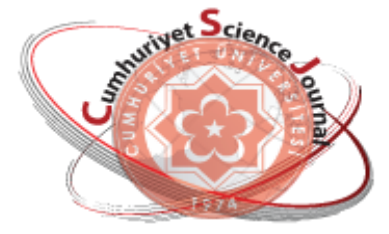

e-ISSN: $2587-246 X$

ISSN: $2587-2680$

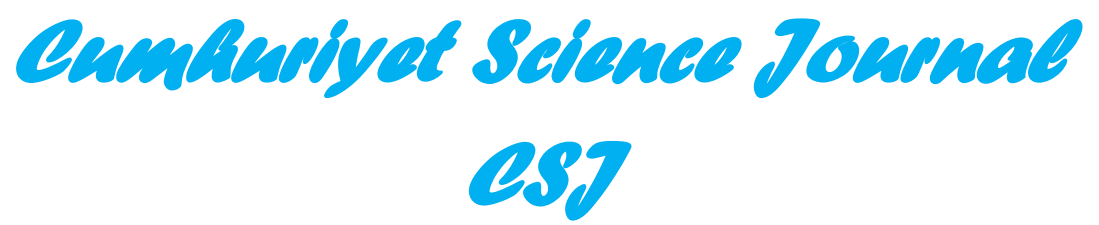

Cumhuriyet Sci. J., Vol.40-2(2019) 317-331

\title{
Interval-Valued Fuzzy Parameterized Intuitionistic Fuzzy Soft Sets and Their Applications
}

\author{
Hüseyin KAMACI ${ }^{1}$ id \\ ${ }^{l}$ Yozgat Bozok University, Faculty of Science and Arts, Department of Mathematics, Yozgat, TURKEY
}

\begin{abstract}
In recent years, the fuzzy sets, interval-valued fuzzy sets, intuitionistic fuzzy sets and soft sets, which offer different perspectives for the structures containing the uncertainties, have attracted the interest of many researchers. Also, the intuitionistic fuzzy soft sets produced by combining the intuitionistic fuzzy sets with the soft sets have been widely studied. In this work, the concept of interval-valued fuzzy parameterized intuitionistic fuzzy soft set (IVFPIFS set) is introduced. This set is the generalization of soft sets, fuzzy soft sets, fuzzy parameterized (fuzzy) soft sets, interval-valued fuzzy parameterized (fuzzy) soft sets, intuitionistic fuzzy soft sets and fuzzy parameterized intuitionistic fuzzy soft sets. For the IVFPIFS sets, basic operations such as complement, union and intersection are defined. Also, the properties of these operations are investigated in detail. Lastly, an algorithm by using the aggregation operators based on the IVFPIFS sets is constructed. The examples are given to verify the feasibility and validity of the proposed algorithm.
\end{abstract}

Keywords: Fuzzy set, Interval-valued fuzzy set, Intuitionistic fuzzy set, Intuitionistic fuzzy soft set, IVFPIFS set [2000] 03E72, 03B52,94D05.

\section{Aralık Değerli Bulanık Parametreli Sezgisel Bulanık Esnek Kümeler ve Uygulamaları}

Özet. Son yıllarda, belirsizlik içeren yapılar için farklı perspektifler sunan bulanık kümeler, aralık değerli bulanık kümeler, sezgisel bulanık kümeler ve esnek kümeler birçok araştırmacının ilgisini çekmiştir. Ayrıca, sezgisel bulanık kümeleri esnek kümelerle birleştirerek oluşturulan sezgisel bulanık esnek kümeler de geniş ölçüde çalışıımıştır. Bu çalışmada, aralık değerli bulanık parametreli sezgisel bulanık esnek küme (ADBPSBE küme) kavramı tanıtılmıştır. Bu küme, esnek kümelerin, bulanık esnek kümelerin, bulanık parametreli (bulanık) esnek kümelerin, aralık değerli bulanık parametreli (bulanık) esnek kümelerin, sezgisel bulanık esnek kümelerin ve bulanık parametreli sezgisel bulanık esnek kümelerin genelleştirilmesidir. ADBPSBE kümeler için tümleyen, birleşim ve kesişim gibi temel işlemler tanımlanmıştır. Ayrıca, bu işlemlerin özellikleri detaylı olarak araştıılmıştır. Son olarak, ADBPSBE kümeler üzerine temellenmiş birleştirme operatörlerini kullanarak bir algoritma oluşturulmuştur. Önerilen algoritmanın uygulanabilirliğini ve geçerliliğini test etmek için örnekler verilmiştir.

Anahtar Kelimeler: Bulanık küme, Aralık değerli bulanık küme, Sezgisel bulanık küme, Sezgisel bulanık esnek küme, ADBPSBE küme [2000] 03E72, 03B52,94D05.

\footnotetext{
* Corresponding author. Email address: huseyin.kamaci@bozok.edu.tr

http://dergipark.gov.tr/csj $\quad$ C2016 Faculty of Science, Sivas Cumhuriyet University
} 


\section{INTRODUCTION}

It is natural to deal with uncertainties and imprecise data in various areas such as economics, environmental science, social science, medical science and engineering. This situation is part of the complexity of the real world. Some kinds of uncertainties are major problems which cannot be dealt with by classical methods. Therefore, the researchers need new mathematical tools to overcome these difficulties. Fuzzy set theory, offered by Zadeh [1], was developed as an important mathematical tool to resolve the uncertainty and ambiguity issues. Sambuc [2] proposed a new approach using $\operatorname{Int}([0,1])$ consisting of all closed subintervals of the interval $[0,1]$ instead of the interval $[0,1]$ in the membership function of the fuzzy set. Moreover, Atanassov [3] defined an intuitionistic fuzzy set which is an extension of the concept of a fuzzy set. Afterwards, Atanassov and Gargov [4] presented the concept of interval-valued intuitionistic fuzzy set developed by taking the interval-valued fuzzy values instead of fuzzy values of membership and non-membership in the intuitionistic fuzzy soft set.

In 1999, Molodtsov [5] introduced the soft set theory which is a general mathematical tool for dealing with uncertain data. In [6-8], the researchers contributed to the operations of soft sets. Furthermore, Aktaş and Çağman [9] focused on the notion of soft group, thus they pioneered the idea of algebraic structures on the soft sets. Immediately after, the algebraic structures such as the soft semiring, soft ring, soft module and soft near-ring were discussed [10-13]. In [14,15], the authors addressed the fuzzification of the parameters of the soft sets. Moreover, the products [16], means [17] and relations [18] of fuzzy parameterized soft sets were derived. Deli and Çağman [19] discussed the soft set, whose parameters have intuitionistic fuzzy weights. Also, Deli and Karataş [20] introduced interval valued intuitionistic fuzzy parameterized soft sets and their some operations. While the operational and structural improvements in the soft sets continued, Çağman et al. [21] initiated a new theory called the fuzzy soft set. Later, they created a selection algorithm using the $f s$-aggregation operator based on the fuzzy soft set. Many researchers aimed to improve and further extend these theories by using the matrix representations of the soft sets and fuzzy soft sets [22-27]. Maji et al. [28] introduced an intuitionistic fuzzy soft set by embedding the idea of the intuitionistic fuzzy set into the soft set. Xu et al. [29] further researched on the intuitionistic fuzzy soft sets. Çağman and Karataş [30] redefined intuitionistic fuzzy soft set by utilizing the concept of fuzzy soft set defined in [21]. Also, they derived new operations for intuitionistic fuzzy soft sets and obtained several propositions and conclusions. In [31], the authors focused on the similarity measure between two intuitionistic fuzzy soft sets. In addition to these developments in the soft set theory, many studies were published about new findings and results on the different types of soft sets such as interval-valued fuzzy soft sets, intuitionistic fuzzy sets and neutrosophic soft sets [32-39].

In this paper, we define IVFPIFS sets and their operations. Also, we give various propositions which are related to the relations and operations on the IVFPIFS sets. Additionally, we present two IVFPIFSaggregation operators and accordingly the aggregate interval-valued intuitionistic fuzzy sets produced by employing these operators. By using new notions, we give an algorithm with illustrative examples.

\section{PRELIMINARIES}

In this section, the concepts of fuzzy set, interval-valued fuzzy set, intuitionistic fuzzy set, intervalvalued intuitionistic fuzzy set, soft set and intuitionistic fuzzy soft set are given.

Definition 2.1. ([1]) Let $A$ be a collection of objects (points) denoted by $a_{i}$. Then, a fuzzy set $W$ in $A$ is defined as

$$
W=\left\{\alpha_{W}\left(a_{i}\right) / a_{i}: a_{i} \in A\right\}
$$


where $\alpha_{W}: A \rightarrow[0,1]$ is called the membership function of $W$. The value $\alpha_{W}\left(a_{i}\right)$ denotes the degree of membership of the element $a_{i} \in A$ into the set $W$.

Definition 2.2. ([2]) Let $A$ be a collection of objects (points) denoted by $a_{i}$. Also, let $\operatorname{Int}([0,1])$ be the set of all closed subintervals of the interval [0,1]. Then, an interval-valued fuzzy set $X$ in $A$ is defined as

$$
X=\left\{\beta_{X}\left(a_{i}\right) / a_{i}: a_{i} \in A\right\}
$$

where $\beta_{X}: A \rightarrow \operatorname{Int}([0,1])$ is called the membership function of $X$ such that $\beta_{X}\left(a_{i}\right)=\left[\underline{\beta_{X}}\left(a_{i}\right), \overline{\beta_{X}}\left(a_{i}\right)\right]$ and $0 \leq \underline{\beta_{X}}\left(a_{i}\right) \leq \overline{\beta_{X}}\left(a_{i}\right) \leq 1$. The values $\underline{\beta_{X}}\left(a_{i}\right)$ and $\overline{\beta_{X}}\left(a_{i}\right)$ denote the lower and upper degrees of membership of the element $a_{i} \in A$ into the set $X$, respectively.

State that the set of all interval-valued fuzzy sets on the set $A$ is denoted by $\operatorname{IVFS}(A)$.

Definition 2.3. ([3]) Let $A$ be a collection of objects (points) denoted by $a_{i}$. Then, an intuitionistic fuzzy set $Y$ in $A$ is defined as

$$
Y=\left\{\prec \gamma_{Y}^{+}\left(a_{i}\right), \gamma_{Y}^{-}\left(a_{i}\right)>/ a_{i}: a_{i} \in A\right\}
$$

where $\gamma_{Y}^{+}, \gamma_{Y}^{-}: A \rightarrow[0,1]$ are respectively called the membership function and the non-membership function of $Y$ with property $0 \leq \gamma_{Y}^{+}\left(a_{i}\right)+\gamma_{Y}^{-}\left(a_{i}\right) \leq 1$. The values $\gamma_{Y}^{+}\left(a_{i}\right)$ and $\gamma_{Y}^{-}\left(a_{i}\right)$ denote the degrees of membership and non-membership of the element $a_{i} \in A$ into the set $Y$, respectively.

The remaining part $\gamma_{Y}^{*}\left(a_{i}\right)=1-\gamma_{Y}^{+}\left(a_{i}\right)-\gamma_{Y}^{-}\left(a_{i}\right)$ is called the indeterministic part of $a_{i} \in A$.

State that the set of all intuitionistic fuzzy sets on the set $A$ is denoted by $\operatorname{IFS}(A)$.

Remark: If $\gamma_{Y}^{+}\left(a_{i}\right)+\gamma_{Y}^{-}\left(a_{i}\right)=1$ for all $a_{i}$ then intuitionistic fuzzy set $Y$ degenerates into a fuzzy set.

Proposition 2.4. ([3]) Let $Y_{1}$ and $Y_{2}$ be two intuitionistic fuzzy sets on $A$. Then,

i) $Y_{1} \subseteq Y_{2} \Leftrightarrow \gamma_{Y_{1}}^{+}\left(a_{i}\right) \leq \gamma_{Y_{2}}^{+}\left(a_{i}\right)$ and $\gamma_{Y_{1}}^{-}\left(a_{i}\right) \geq \gamma_{Y_{2}}^{-}\left(a_{i}\right)$ for all $a_{i} \in A$.

ii) $Y_{1} \cap Y_{2}=\left\{\prec \inf \left\{\gamma_{Y_{1}}^{+}\left(a_{i}\right), \gamma_{Y_{2}}^{+}\left(a_{i}\right)\right\}, \sup \left\{\gamma_{Y_{1}}^{-}\left(a_{i}\right), \gamma_{Y_{2}}^{-}\left(a_{i}\right)\right\}>/ a_{i}: a_{i} \in A\right\}$.

iii) $Y_{1} \cup Y_{2}=\left\{\prec \sup \left\{\gamma_{Y_{1}}^{+}\left(a_{i}\right), \gamma_{Y_{2}}^{+}\left(a_{i}\right)\right\}, \inf \left\{\gamma_{Y_{1}}^{-}\left(a_{i}\right), \gamma_{Y_{2}}^{-}\left(a_{i}\right)\right\}>/ a_{i}: a_{i} \in A\right\}$.

iv) $Y_{1}^{c}=\left\{\prec \gamma_{Y_{1}}^{-}\left(a_{i}\right), \gamma_{Y_{1}}^{+}\left(a_{i}\right)>/ a_{i}: a_{i} \in A\right\}$.

Definition 2.5. ([4]) Let $A$ be a collection of objects (points) denoted by $a_{i}$. Also, let $\operatorname{Int}([0,1])$ be the set of all closed subintervals of the interval [0,1]. Then, an interval-valued intuitionistic fuzzy set $Z$ in $A$ is defined as

$$
Z=\left\{\prec \gamma_{Z}^{+}, \gamma_{Z}^{-}>/ a_{i}: a_{i} \in A\right\}
$$

where $\gamma_{Z}^{+}=\left[\underline{\gamma_{Z}^{+}}\left(a_{i}\right), \overline{\gamma_{Z}^{+}}\left(a_{i}\right)\right], \gamma_{\bar{Z}}^{-}=\left[\underline{\gamma_{\bar{Z}}}\left(a_{i}\right), \overline{\gamma_{Z}^{-}}\left(a_{i}\right)\right]: A \rightarrow \operatorname{Int}([0,1])$ are respectively called the membership function and the non-membership function of $Z$ with property $0 \leq \overline{\gamma_{Z}^{+}}\left(a_{i}\right)+\overline{\gamma_{Z}^{-}}\left(a_{i}\right) \leq 1$ and $0 \leq \underline{\gamma_{Z}^{+}}\left(a_{i}\right)+\underline{\gamma_{Z}^{-}}\left(a_{i}\right) \leq 1$. Here, the values $\underline{\gamma_{Z}^{+}}\left(a_{i}\right)$ and $\overline{\gamma_{Z}^{+}}\left(a_{i}\right)$ denote the lower and upper degrees of membership of the element $a_{i} \in A$, and also the values $\underline{\gamma_{Z}^{-}}\left(a_{i}\right)$ and $\overline{\gamma_{Z}^{-}}\left(a_{i}\right)$ denote the lower and upper degrees of non-membership of the element $a_{i} \in A$, respectively. 
State that the set of all interval-valued intuitionistic fuzzy sets on the set $A$ is denoted by $\operatorname{IVIFS}(A)$.

Note: If we use notations $\gamma_{i}^{+}$and $\gamma_{i}^{-}$instead of $\gamma_{Z}^{+}\left(a_{i}\right)$ and $\gamma_{Z}^{-}\left(a_{i}\right)$ for $a_{i} \in A$, then $\gamma_{i}=\prec \gamma_{i}^{+}, \gamma_{i}^{-}>=$ $\prec\left[\underline{\gamma_{i}^{+}}, \overline{\gamma_{i}^{+}}\right],\left[\underline{\gamma_{i}^{-}}, \overline{\gamma_{i}^{-}}\right]>$denotes interval-valued intuitionistic fuzzy value (shortly, IVIF value) of $a_{i} \in A$.

Definition 2.6. ([40,41]) Let $\gamma_{i}=<\gamma_{i}^{+}, \gamma_{i}^{-}>=<\left[\underline{\gamma_{i}^{+}}, \overline{\gamma_{i}^{+}}\right],\left[\underline{\gamma_{i}^{-}}, \overline{\gamma_{i}^{-}}\right]>$be the IVIF value of $a_{i} \in A$. Then, the score function and accuracy function of $\gamma_{i}$ are respectively defined as follows:

$$
\begin{aligned}
& \operatorname{Scr}\left(\gamma_{i}\right)=\frac{1}{2}\left(\underline{\gamma_{i}^{+}}-\underline{\gamma_{i}^{-}}+\overline{\gamma_{i}^{+}}-\overline{\gamma_{i}^{-}}\right), \\
& \operatorname{Acr}\left(\gamma_{i}\right)=\frac{1}{2}\left(\underline{\gamma_{i}^{+}}+\underline{\gamma_{i}^{-}}+\overline{\gamma_{i}^{+}}+\overline{\gamma_{i}^{-}}\right) .
\end{aligned}
$$

To compare two IVIF values $\gamma_{1}$ and $\gamma_{2}$, Tan [40] and $\mathrm{Xu}$ [41] introduced a simple method as follows:

(1) If $\operatorname{Scr}\left(\gamma_{1}\right)<\operatorname{Scr}\left(\gamma_{2}\right)$ then $\gamma_{1}<\gamma_{2}$

(2) If $\operatorname{Scr}\left(\gamma_{1}\right)=\operatorname{Scr}\left(\gamma_{2}\right)$ then

(i) if $\operatorname{Acr}\left(\gamma_{1}\right)<\operatorname{Acr}\left(\gamma_{2}\right)$ then $\gamma_{1}<\gamma_{2}$

(ii) if $\operatorname{Acr}\left(\gamma_{1}\right)=\operatorname{Acr}\left(\gamma_{2}\right)$ then $\gamma_{1}=\gamma_{2}$.

Definition 2.7. ([41]) Let $\gamma_{1}=<\left[\underline{\gamma_{1}^{+}}, \overline{\gamma_{1}^{+}}\right],\left[\underline{\gamma_{1}^{-}}, \overline{\gamma_{1}^{-}}\right]>$and $\gamma_{2}=<\left[\underline{\gamma_{2}^{+}}, \overline{\gamma_{2}^{+}}\right],\left[\underline{\gamma_{2}^{-}}, \overline{\gamma_{2}^{-}}\right]>$be two IVIF values. Then, the operations $\oplus$ and $\otimes$ for $\gamma_{1}$ and $\gamma_{2}$ are respectively defined as follows:

a) $\gamma_{1} \oplus \gamma_{2}=<\left[\underline{\gamma_{1}^{+}}+\underline{\gamma_{2}^{+}}-\underline{\gamma_{1}^{+}} \cdot \underline{\gamma_{2}^{+}}, \overline{\gamma_{1}^{+}}+\overline{\gamma_{2}^{+}}-\overline{\gamma_{1}^{+}} \cdot \overline{\gamma_{2}^{+}}\right],\left[\underline{\gamma_{1}^{-}} \cdot \underline{\gamma_{2}^{-}}, \overline{\gamma_{1}^{-}} \cdot \overline{\gamma_{2}^{-}}\right]>$.

b) $\gamma_{1} \otimes \gamma_{2}=<\left[\underline{\gamma_{1}^{+}} \cdot \underline{\gamma_{2}^{+}}, \overline{\gamma_{1}^{+}} \cdot \overline{\gamma_{2}^{+}}\right],\left[\underline{\gamma_{1}^{-}}+\underline{\gamma_{2}^{-}}-\underline{\gamma_{1}^{-}} \cdot \underline{\gamma_{2}^{-}}, \overline{\gamma_{1}^{-}}+\overline{\gamma_{2}^{-}}-\overline{\gamma_{1}^{-}} \cdot \overline{\gamma_{2}^{-}}\right] \succ$.

Definition 2.8. ([5,42]) Let $U$ be a universal set and $P(U)$ be the power set of $U$. Also, let $\mathcal{E}$ be the set of parameters and $\mathcal{A} \subseteq \mathcal{E}$. Then, a soft set $\mathcal{F}_{\mathcal{A}}$ on $U$ is a set of ordered pairs

$$
\mathcal{F}_{\mathcal{A}}=\left\{\left(\varepsilon_{j}, f_{\mathcal{A}}\left(\varepsilon_{j}\right)\right): \varepsilon_{j} \in \mathcal{E}, f_{\mathcal{A}}\left(\varepsilon_{j}\right) \in P(U)\right\}
$$

where the function $f_{\mathcal{A}}: \mathcal{E} \rightarrow P(U)$ such that $f_{\mathcal{A}}\left(\varepsilon_{j}\right)=\emptyset$ if $\varepsilon_{j} \notin \mathcal{A}$. Also, $f_{\mathcal{A}}$ is called an approximate function of the soft set $\mathcal{F}_{\mathcal{A}}$.

Definition 2.9. ([30]) Let $U$ be a universal set. Also, let $\mathcal{E}$ be the set of parameters and $\mathcal{A} \subseteq \mathcal{E}$. Then, an intuitionistic fuzzy soft set (shortly, IFS set) $\Psi_{\mathcal{A}}$ on $U$ is a set of ordered pairs

$$
\Psi_{\mathcal{A}}=\left\{\left(\varepsilon_{j}, \psi_{\mathcal{A}}\left(\varepsilon_{j}\right)\right): \varepsilon_{j} \in \mathcal{E}, \psi_{\mathcal{A}}\left(\varepsilon_{j}\right) \in \operatorname{IFS}(U)\right\}
$$

where the function $\psi_{\mathcal{A}}: \mathcal{E} \rightarrow \operatorname{IFS}(U)$ such that $\psi_{\mathcal{A}}\left(\varepsilon_{j}\right)=\emptyset$ if $\varepsilon_{j} \notin \mathcal{A}$. Also, $\psi_{\mathcal{A}}$ is called an approximate function of the intuitionistic fuzzy soft set $\Psi_{\mathcal{A}}$.

State that the set of all intuitionistic fuzzy soft sets on the set $U$ is denoted by $\operatorname{IFSS}(U)$. 


\section{INTERVAL-VALUED FUZZY PARAMETERIZED INTUITIONISTIC FUZZY SOFT SET}

In this section, the interval-valued fuzzy parameterized intuitionistic fuzzy soft set (shortly, IVFPIFS set) is defined. Also, it is given some specific findings and results for the IVFPIFS sets.

Definition 3.1. Let $U$ be a universal set and $\mathcal{E}$ be the set of parameters. Also, let $X$ be a interval-valued fuzzy set over $\mathcal{E}$ with the membership function $\beta_{X}: \mathcal{E} \rightarrow \operatorname{Int}([0,1])$. Then, an interval-valued fuzzy parameterized intuitionistic fuzzy soft set (IVFPIFS set) $\Psi_{X}$ on $U$ is a set of ordered pairs

$$
\Psi_{X}=\left\{\left(\beta_{X}\left(\varepsilon_{j}\right) / \varepsilon_{j}, \psi_{X}\left(\varepsilon_{j}\right)\right): \varepsilon_{j} \in \mathcal{E}, \psi_{X}\left(\varepsilon_{j}\right) \in \operatorname{IFS}(U)\right\}
$$

where the function $\psi_{X}: \mathcal{E} \rightarrow \operatorname{IFS}(U)$ such that $\psi_{X}\left(\varepsilon_{j}\right)=\emptyset$ if $\beta_{X}\left(\varepsilon_{j}\right)=\left[\underline{\beta_{X}}\left(\varepsilon_{j}\right), \overline{\beta_{X}}\left(\varepsilon_{j}\right)\right]=[0,0]$.

State that the set of all interval-valued fuzzy parameterized intuitionistic fuzzy soft sets on $U$ is denoted by $\operatorname{IVFPIFSS}(U)$.

Example 3.2. Let us assume that $U=\left\{u_{1}, u_{2}, u_{3}, u_{4}\right\}$ is the set of cars which can be purchased in a car showroom. To evaluate these cars, there may be five parameters $\varepsilon=\left\{\varepsilon_{1}, \varepsilon_{2}, \varepsilon_{3}, \varepsilon_{4}, \varepsilon_{5}\right\}$, where $\varepsilon_{j}, j=$ $1,2,3,4,5$ stand for "equipped", "speedy", "sport", "cheap" and "modern", resp. If it is taken $X_{1}=$ $\left\{[0.2,0.6] / \varepsilon_{1},[0.55,0.75] / \varepsilon_{2},[0.1,0.85] / \varepsilon_{3},[0,1] / \varepsilon_{4},[0.2,0.25] / \varepsilon_{5}\right\} \quad$ and $\quad \psi_{X_{1}}\left(\varepsilon_{1}\right)=\{<0.4,0.3 \succ$ $\left./ u_{1},<0.7,0.2>/ u_{3},<0.11,0.7>/ u_{4}\right\}, \quad \psi_{X_{1}}\left(\varepsilon_{2}\right)=\left\{<0.6,0.1>/ u_{2}\right\}, \quad \psi_{X_{1}}\left(\varepsilon_{3}\right)=<0.5,0.25>/ U$, $\psi_{X_{1}}\left(\varepsilon_{4}\right)=\left\{\prec 0.6,0.35>/ u_{1}, \prec 0.4,0>/ u_{2}, \prec 0.4,0.47>/ u_{4}\right\}, \psi_{X_{1}}\left(\varepsilon_{5}\right)=\left\{\prec 0.5,0.4>/ u_{3}\right\}$, then the IVFPIFS set is written as

$$
\begin{aligned}
\Psi_{X_{1}}=\{ & \left([0.2,0.6] / \varepsilon_{1},\left\{\prec 0.4,0.3>/ u_{1}, \prec 0.7,0.2>/ u_{3}, \prec 0.11,0.7>/ u_{4}\right\}\right), \\
& \left([0.55,0.75] / \varepsilon_{2},\left\{\prec 0.6,0.1>/ u_{2}\right\}\right),\left([0.1,0.85] / \varepsilon_{3}, \prec 0.5,0.25>/ U\right), \\
& \left([0,1] / \varepsilon_{4},\left\{\prec 0.6,0.35>/ u_{1}, \prec 0.4,0>/ u_{2}, \prec 0.4,0.47>/ u_{4}\right\}\right), \\
& \left.\left([0.2,0.25] / \varepsilon_{5},\left\{<0.5,0.4>/ u_{3}\right\}\right)\right\} .
\end{aligned}
$$

Definition 3.3. Let $\Psi_{X} \in \operatorname{IVFPIFSS}(U)$. Then, $\Psi_{X}$ is called

a) an empty IVFPIFS set if it is denoted and defined as

$$
\Psi_{\bar{\varnothing}}=\left\{\left([0,0] / \varepsilon_{j},\left\{<0,1>/ u_{i}: u_{i} \in U\right\}\right): \varepsilon_{j} \in \mathcal{E}\right\}
$$

b) a universal IVFPIFS set if it is denoted and defined as

$$
\Psi_{\bar{\varepsilon}}=\left\{\left([1,1] / \varepsilon_{j},\left\{<1,0>/ u_{i}: u_{i} \in U\right\}\right): \varepsilon_{j} \in \mathcal{E}\right\}
$$

Definition 3.4. Let $\Psi_{X_{1}}, \Psi_{X_{2}} \in \operatorname{IVFPIFSS}(U)$. Then,

a) $\Psi_{X_{1}}$ is an interval-valued fuzzy parameterized intuitionistic fuzzy soft subset of $\Psi_{X_{2}}$ if and only if $\underline{\beta_{X_{1}}}\left(\varepsilon_{j}\right) \leq \underline{\beta_{X_{2}}}\left(\varepsilon_{j}\right), \overline{\beta_{X_{1}}}\left(\varepsilon_{j}\right) \leq \overline{\beta_{X_{2}}}\left(\varepsilon_{j}\right)$ and $\psi_{X_{1}}\left(\varepsilon_{j}\right) \subseteq \psi_{X_{2}}\left(\varepsilon_{j}\right)$ for all $\varepsilon_{j} \in \mathcal{E}$. This is denoted by $\overline{\Psi_{X_{1}}} \widetilde{\widetilde{\Xi}} \Psi_{X_{2}}$.

b) $\Psi_{X_{1}}$ and $\Psi_{X_{2}}$ are interval-valued fuzzy parameterized intuitionistic fuzzy soft equal if and only if $\underline{\beta_{X_{1}}}\left(\varepsilon_{j}\right)=\underline{\beta_{X_{2}}}\left(\varepsilon_{j}\right), \overline{\beta_{X_{1}}}\left(\varepsilon_{j}\right)=\overline{\beta_{X_{2}}}\left(\varepsilon_{j}\right)$ and $\psi_{X_{1}}\left(\varepsilon_{j}\right) \subseteq \psi_{X_{2}}\left(\varepsilon_{j}\right), \psi_{X_{1}}\left(\varepsilon_{j}\right) \supseteq \psi_{X_{2}}\left(\varepsilon_{j}\right)$ for all $\varepsilon_{j} \in \mathcal{E}$. This is denoted by $\Psi_{X_{1}}=\Psi_{X_{2}}$. 
Example 3.5. We consider the IVFPIFS set $\Psi_{X_{1}}$ in Example 3.2. Also, we take the following IVFPIFS set, for $X_{2}=\left\{[0,0.2] / \varepsilon_{1},[0.3,0.5] / \varepsilon_{2},[0.1,0.85] / \varepsilon_{3},[0,0.7] / \varepsilon_{4},[0.1,0.2] / \varepsilon_{5}\right\}$,

$$
\begin{aligned}
\Psi_{X_{2}}=\{ & \left([0,0.2] / \varepsilon_{1},\left\{<0.2,05>/ u_{1},<0.11,0.8>/ u_{4}\right\}\right),\left([0.3,0.5] / \varepsilon_{2},\left\{<0.3,0.65>/ u_{2}\right\}\right), \\
& \left.\left([0.1,0.85] / \varepsilon_{3},\left\{<0.22,0.51>/ u_{2},<0.37,0.25>/ u_{3}\right\},<0.45,0.65>/ u_{4}\right\}\right), \\
& \left.\left([0,0.7] / \varepsilon_{4},\left\{<0.3,0.5>/ u_{1}, \prec 0.3,0>/ u_{2}\right\}\right),\left([0.1,0.2] / \varepsilon_{5},\left\{<0.1,0.8>/ u_{3}\right\}\right)\right\} .
\end{aligned}
$$

Then, we can say that $\Psi_{X_{2}} \widetilde{\subseteq} \Psi_{X_{1}}$.

Proposition 3.6. Let $\Psi_{X_{1}}, \Psi_{X_{2}}, \Psi_{X_{3}} \in \operatorname{IVFPIFSS}(U)$. Then,

i) $\Psi_{X_{1}} \widetilde{\subseteq} \Psi_{\bar{\varepsilon}}$.

ii) $\Psi_{\bar{\emptyset}} \widetilde{\simeq} \Psi_{\bar{\varepsilon}}$.

iii) $\Psi_{X_{1}} \widetilde{\simeq} \Psi_{X_{1}}$.

iv) $\Psi_{X_{1}} \widetilde{\subseteq} \Psi_{X_{2}}$ and $\Psi_{X_{2}} \widetilde{\subseteq} \Psi_{X_{1}} \Leftrightarrow \Psi_{X_{1}}=\Psi_{X_{2}}$.

v) $\Psi_{X_{1}} \widetilde{\simeq} \Psi_{X_{2}}$ and $\Psi_{X_{2}} \widetilde{\simeq} \Psi_{X_{3}} \Rightarrow \Psi_{X_{1}} \widetilde{\subseteq} \Psi_{X_{3}}$.

vi) $\Psi_{X_{1}}=\Psi_{X_{2}}$ and $\Psi_{X_{2}}=\Psi_{X_{3}} \Rightarrow \Psi_{X_{1}}=\Psi_{X_{3}}$.

Proof. The proofs are straightforward, hence omitted.

Definition 3.7. Let $\Psi_{X} \in \operatorname{IVFPIFSS}(U)$. Then, the complement of $\Psi_{X}$, symbolized by $\Psi_{X}^{c}$, is defined by

$$
\Psi_{X}^{c}=\left\{\left(\beta_{X} \tilde{c}\left(\varepsilon_{j}\right) / \varepsilon_{j}, \psi_{X} \tilde{c}\left(\varepsilon_{j}\right)\right): \varepsilon_{j} \in \mathcal{E}, \psi_{X^{\tilde{c}}}\left(\varepsilon_{j}\right) \in \operatorname{IFS}(U)\right\}
$$

where $\beta_{X} \tilde{c}\left(\varepsilon_{j}\right)=1-\beta_{X}\left(\varepsilon_{j}\right)=\left[1-\overline{\beta_{X}}\left(\varepsilon_{j}\right), 1-\underline{\beta_{X}}\left(\varepsilon_{j}\right)\right]$ and $\psi_{X} \tilde{c}\left(\varepsilon_{j}\right)$ is the complement of intuitionistic fuzzy set $\psi_{X}\left(\varepsilon_{j}\right)$.

Example 3.8. We consider the IVFPIFS set $\Psi_{X_{1}}$ given in Example 3.2. The complement of the IVFPIFS set $\Psi_{X_{1}}$ is

$$
\begin{aligned}
\Psi_{X_{1}}^{c}=\{ & \left([0.4,0.8] / \varepsilon_{1},\left\{<0.3,0.4>/ u_{1}, \prec 1,0>/ u_{2}, \prec 0.2,0.7>/ u_{3},<0.7,0.11>/ u_{4}\right\}\right), \\
& \left([0.25,0.45] / \varepsilon_{2},\left\{<1,0>/ u_{1},<0.1,0.6>/ u_{2},<1,0>/ u_{3}, \prec 1,0>/ u_{4}\right)\right\}, \\
& \left([0.15,0.9] / \varepsilon_{3}, \prec 0.25,0.5>/ U\right), \\
& \left([0,1] / \varepsilon_{4},\left\{<0.35,0.6>/ u_{1},<0,0.4>/ u_{2},<1,0>/ u_{3}, \prec 0.47,0.4>/ u_{4}\right\}\right), \\
& \left.\left([0.75,0.8] / \varepsilon_{5},\left\{<1,0>/ u_{1}, \prec 1,0>/ u_{2},<0.4,0.5>/ u_{3}\right\}\right),<1,0>/ u_{4}\right\} .
\end{aligned}
$$

Proposition 3.9. Let $\Psi_{X} \in \operatorname{IVFPIFSS}(U)$.
i) $\Psi_{\overline{\mathcal{E}}}^{c}=\Psi_{\bar{\emptyset}}$.
ii) $\Psi_{\bar{\emptyset}}^{c}=\Psi_{\bar{\varepsilon}}$.
iii) $\left(\Psi_{X}^{c}\right)^{c}=\Psi_{X}$.

Proof. The proofs are trivial.

Definition 3.10. Let $\Psi_{X_{1}}, \Psi_{X_{2}} \in \operatorname{IVFPIFSS}(U)$. Then, the union of $\Psi_{X_{1}}$ and $\Psi_{X_{2}}$, symbolized by $\Psi_{X_{1}} \widetilde{\square} \Psi_{X_{2}}$, is defined by

$$
\Psi_{X_{1}} \widetilde{\square} \Psi_{X_{2}}=\left\{\left(\beta_{X_{1}} \widetilde{\square} X_{2}\left(\varepsilon_{j}\right) / \varepsilon_{j}, \psi_{X_{1} \widetilde{\square} X_{2}}\left(\varepsilon_{j}\right)\right): \varepsilon_{j} \in \mathcal{E}, \psi_{X_{1} \widetilde{\square} X_{2}}\left(\varepsilon_{j}\right) \in I F S(U)\right\}
$$


where $\beta_{X_{1} \widetilde{\square} X_{2}}\left(\varepsilon_{j}\right)=\sup \left[\beta_{X_{1}}\left(\varepsilon_{j}\right), \beta_{X_{2}}\left(\varepsilon_{j}\right)\right]=\left[\sup \left\{\underline{\beta_{X_{1}}}\left(\varepsilon_{j}\right), \underline{\beta_{X_{2}}}\left(\varepsilon_{j}\right)\right\}, \sup \left\{\overline{\beta_{X_{1}}}\left(\varepsilon_{j}\right), \overline{\beta_{X_{2}}}\left(\varepsilon_{j}\right)\right\}\right]$ and $\psi_{X_{1} \widetilde{\square} X_{2}}\left(\varepsilon_{j}\right)=\psi_{X_{1}}\left(\varepsilon_{j}\right) \cup \psi_{X_{2}}\left(\varepsilon_{j}\right)$.

Example 3.11. Let's consider the IVFPIFS set $\Psi_{X_{1}}$ in Example 3.2. Also, we take the IVFPIFS set

$$
\begin{aligned}
\Psi_{X_{2}}=\{ & \left([0.3,0.7] / \varepsilon_{1},\left\{<0.5,0.4>/ u_{1},<0.1,0.7>/ u_{4}\right\}\right), \\
& \left([0.55,0.8] / \varepsilon_{2},\left\{<0.2,0.75>/ u_{1},<0.35,0.35>/ u_{3}\right\}\right), \\
& \left([0.3,0.9] / \varepsilon_{3},\left\{<0.6,0.3>/ u_{1}, \prec 0.6,0.2>/ u_{2}\right\}\right), \\
& \left([0.1,0.5] / \varepsilon_{4},\left\{<0.5,0.35>/ u_{1},<0.2,0.4>/ u_{2},<0.5,0.47>/ u_{4}\right\}\right), \\
& \left.\left([0.2,0.25] / \varepsilon_{5},\left\{<0.1,0.5>/ u_{3}\right\}\right)\right\} .
\end{aligned}
$$

Then, the union of the IVFPIFS sets $\Psi_{X_{1}}$ and $\Psi_{X_{2}}$ is

$$
\begin{aligned}
\Psi_{X_{1}} \widetilde{\square} \Psi_{X_{2}}=\{ & \left([0.3,0.7] / \varepsilon_{1},\left\{<0.5,0.3>/ u_{1},<0.7,0.2>/ u_{3}, \prec 0.11,0.7>/ u_{4}\right\}\right), \\
& \left([0.55,0.8] / \varepsilon_{2},\left\{<0.2,0.75>/ u_{1},<0.6,0.1>/ u_{2},<0.35,0.35>/ u_{3}\right\}\right), \\
& \left([0.3,0.9] / \varepsilon_{3},\left\{<0.6,0.25>/ u_{1},<0.6,0.2>/ u_{2},<0.5,0.25>/ u_{3},<0.5,0.25>/ u_{4}\right\}\right), \\
& \left([0.1,1] / \varepsilon_{4},\left\{<0.6,0.35>/ u_{1},<0.4,0>/ u_{2},<0.5,0.47>/ u_{4}\right\}\right), \\
& \left.\left([0.2,0.25] / \varepsilon_{5},\left\{<0.5,0.4>/ u_{3}\right\}\right)\right\} .
\end{aligned}
$$

Proposition 3.12. Let $\Psi_{X_{1}}, \Psi_{X_{2}}, \Psi_{X_{3}} \in \operatorname{IVFPIFSS}(U)$. Then,

i) $\Psi_{X_{1}} \widetilde{\sqcup} \Psi_{X_{1}}=\Psi_{X_{1}}$.

ii) $\Psi_{X_{1}} \widetilde{\cup} \Psi_{\bar{\emptyset}}=\Psi_{X_{1}}$.

iii) $\Psi_{X_{1}} \widetilde{\cup} \Psi_{\bar{\varepsilon}}=\Psi_{\overline{\mathcal{\varepsilon}}}$.

iv) $\Psi_{X_{1}} \widetilde{\square} \Psi_{X_{2}}=\Psi_{X_{2}} \widetilde{\sqcup} \Psi_{X_{1}}$.

v) $\Psi_{X_{1}} \widetilde{\sqcup}\left(\Psi_{X_{2}} \widetilde{\sqcup} \Psi_{X_{3}}\right)=\left(\Psi_{X_{1}} \widetilde{\sqcup} \Psi_{X_{2}}\right) \widetilde{\sqcup} \Psi_{X_{3}}$.

Proof. The proofs of (i)-(iv) are trivial, therefore omitted.

v) For all $\varepsilon_{j} \in \mathcal{E}$,

$$
\begin{aligned}
& \beta_{X_{1} \widetilde{\square}\left(X_{2} \widetilde{\square} X_{3}\right)}\left(\varepsilon_{j}\right)=\sup \left[\beta_{X_{1}}\left(\varepsilon_{j}\right), \beta_{X_{2} \widetilde{\square} X_{3}}\left(\varepsilon_{j}\right)\right] \\
& =\sup \left[\beta_{X_{1}}\left(\varepsilon_{j}\right), \sup \left[\beta_{X_{2}}\left(\varepsilon_{j}\right), \beta_{X_{3}}\left(\varepsilon_{j}\right)\right]\right] \\
& =\sup \left[\beta_{X_{1}}\left(\varepsilon_{j}\right),\left[\sup \left\{\underline{\beta_{X_{2}}}\left(\varepsilon_{j}\right), \underline{\beta_{X_{3}}}\left(\varepsilon_{j}\right)\right\}, \sup \left\{\overline{\beta_{X_{2}}}\left(\varepsilon_{j}\right), \overline{\beta_{X_{3}}}\left(\varepsilon_{j}\right)\right\}\right]\right] \\
& =\left[\sup \left\{\underline{\beta_{X_{1}}}\left(\varepsilon_{j}\right), \sup \left\{\underline{\beta_{X_{2}}}\left(\varepsilon_{j}\right), \underline{\beta_{X_{3}}}\left(\varepsilon_{j}\right)\right\}\right\}, \sup \left\{\overline{\beta_{X_{1}}}\left(\varepsilon_{j}\right), \sup \left\{\overline{\beta_{X_{2}}}\left(\varepsilon_{j}\right), \overline{\beta_{X_{3}}}\left(\varepsilon_{j}\right)\right\}\right\}\right] \\
& =\left[\sup \left\{\sup \left\{\underline{\beta_{X_{1}}}\left(\varepsilon_{j}\right), \underline{\beta_{X_{2}}}\left(\varepsilon_{j}\right)\right\}, \underline{\beta_{X_{3}}}\left(\varepsilon_{j}\right)\right\}, \sup \left\{\sup \left\{\overline{\beta_{X_{1}}}\left(\varepsilon_{j}\right), \overline{\beta_{X_{2}}}\left(\varepsilon_{j}\right)\right\}, \overline{\beta_{X_{3}}}\left(\varepsilon_{j}\right)\right\}\right] \\
& \left.=\sup \left[\sup \left\{\underline{\beta_{X_{1}}}\left(\varepsilon_{j}\right), \underline{\beta_{X_{2}}}\left(\varepsilon_{j}\right)\right\}, \sup \left\{\overline{\beta_{X_{1}}}\left(\varepsilon_{j}\right), \overline{\beta_{X_{2}}}\left(\varepsilon_{j}\right)\right\}\right], \beta_{X_{3}}\left(\varepsilon_{j}\right)\right] \\
& =\sup \left[\sup \left[\beta_{X_{1}}\left(\varepsilon_{j}\right), \beta_{X_{2}}\left(\varepsilon_{j}\right)\right], \beta_{X_{3}}\left(\varepsilon_{j}\right)\right] \\
& =\sup \left[\beta_{X_{1} \widetilde{\square} X_{2}}\left(\varepsilon_{j}\right), \beta_{X_{3}}\left(\varepsilon_{j}\right)\right] \\
& =\beta_{\left(X_{1} \widetilde{\square} X_{2}\right) \widetilde{\sqcup} X_{3}}\left(\varepsilon_{j}\right), \\
& \psi_{X_{1} \widetilde{\sqcup}\left(X_{2} \widetilde{\square} X_{3}\right)}\left(\varepsilon_{j}\right)=\psi_{X_{1}}\left(\varepsilon_{j}\right) \cup \psi_{X_{2} \widetilde{\square} X_{3}}\left(\varepsilon_{j}\right) \\
& =\psi_{X_{1}}\left(\varepsilon_{j}\right) \cup\left(\psi_{X_{2}}\left(\varepsilon_{j}\right) \cup \psi_{X_{3}}\left(\varepsilon_{j}\right)\right)
\end{aligned}
$$




$$
\begin{aligned}
& =\left(\psi_{X_{1}}\left(\varepsilon_{j}\right) \cup \psi_{X_{2}}\left(\varepsilon_{j}\right)\right) \cup \psi_{X_{3}}\left(\varepsilon_{j}\right) \\
& =\psi_{X_{1} \widetilde{\square} X_{2}}\left(\varepsilon_{j}\right) \cup \psi_{X_{3}}\left(\varepsilon_{j}\right) \\
& =\psi_{\left(X_{1} \widetilde{\square} X_{2}\right) \widetilde{\sqcup} X_{3}}\left(\varepsilon_{j}\right) .
\end{aligned}
$$

Hence, we obtain that $\Psi_{X_{1}} \widetilde{\sqcup}\left(\Psi_{X_{2}} \widetilde{\sqcup} \Psi_{X_{3}}\right)=\left(\Psi_{X_{1}} \widetilde{\sqcup} \Psi_{X_{2}}\right) \widetilde{\sqcup} \Psi_{X_{3}}$.

Definition 3.13. Let $\Psi_{X_{1}}, \Psi_{X_{2}} \in \operatorname{IVFPIFSS}(U)$. Then, the intersection of $\Psi_{X_{1}}$ and $\Psi_{X_{2}}$, symbolized by $\Psi_{X_{1}} \widetilde{\Pi} \Psi_{X_{2}}$, is defined by

$$
\Psi_{X_{1}} \widetilde{\Pi} \Psi_{X_{2}}=\left\{\left(\beta_{X_{1} \widetilde{\Pi} X_{2}}\left(\varepsilon_{j}\right) / \varepsilon_{j}, \psi_{X_{1} \widetilde{\Pi} X_{2}}\left(\varepsilon_{j}\right)\right): \varepsilon_{j} \in \mathcal{E}, \psi_{X_{1} \widetilde{\Pi} X_{2}}\left(\varepsilon_{j}\right) \in \operatorname{IFS}(U)\right\}
$$

where $\beta_{X_{1} \widetilde{\Pi} X_{2}}\left(\varepsilon_{j}\right)=\inf \left[\beta_{X_{1}}\left(\varepsilon_{j}\right), \beta_{X_{2}}\left(\varepsilon_{j}\right)\right]=\left[\inf \left\{\underline{\beta_{X_{1}}}\left(\varepsilon_{j}\right), \underline{\beta_{X_{2}}}\left(\varepsilon_{j}\right)\right\}, \inf \left\{\overline{\beta_{X_{1}}}\left(\varepsilon_{j}\right), \overline{\beta_{X_{2}}}\left(\varepsilon_{j}\right)\right\}\right]$ and $\psi_{X_{1} \widetilde{\Pi} X_{2}}\left(\varepsilon_{j}\right)=\psi_{X_{1}}\left(\varepsilon_{j}\right) \cap \psi_{X_{2}}\left(\varepsilon_{j}\right)$.

Example 3.14. We take the IVFPIFS set $\Psi_{X_{1}}$ in Example 3.2 and the IVFPIFS set $\Psi_{X_{2}}$ in Example 3.11. Then, the intersection of the IVFPIFS sets $\Psi_{X_{1}}$ and $\Psi_{X_{2}}$ is

$$
\begin{aligned}
\Psi_{X_{1}} \widetilde{\Pi} \Psi_{X_{2}}= & \left\{\left([0.2,0.6] / \varepsilon_{1},\left\{<0.4,0.4>/ u_{1}, \prec 0.1,0.7>/ u_{4}\right\}\right),\left([0.55,0.75] / \varepsilon_{2}, \emptyset\right),\right. \\
& \left([0.1,0.85] / \varepsilon_{3},\left\{<0.5,0.3>/ u_{1}, \prec 0.5,0.25>/ u_{2}\right\}\right), \\
& \left([0,0.5] / \varepsilon_{4},\left\{<0.5,0.35>/ u_{1},<0.2,0.4>/ u_{2}, \prec 0.4,0.47>/ u_{4}\right\}\right), \\
& \left.\left([0.2,0.25] / \varepsilon_{5},\left\{<0.1,0.5>/ u_{3}\right\}\right)\right\} .
\end{aligned}
$$

Proposition 3.15. Let $\Psi_{X_{1}}, \Psi_{X_{2}}, \Psi_{X_{3}} \in \operatorname{IVFPIFSS}(U)$. Then,

i) $\Psi_{X_{1}} \widetilde{\Pi} \Psi_{X_{1}}=\Psi_{X_{1}}$.

ii) $\Psi_{X_{1}} \widetilde{\Pi} \Psi_{\bar{\emptyset}}=\Psi_{\bar{\emptyset}}$.

iii) $\Psi_{X_{1}} \widetilde{\Pi} \Psi_{\bar{\varepsilon}}=\Psi_{X_{1}}$.

iv) $\Psi_{X_{1}} \widetilde{\Pi} \Psi_{X_{2}}=\Psi_{X_{2}} \bar{\Pi} \Psi_{X_{1}}$.

v) $\left(\Psi_{X_{1}} \widetilde{\Pi} \Psi_{X_{2}}\right) \widetilde{\Pi} \Psi_{X_{3}}=\Psi_{X_{1}} \widetilde{\Pi}\left(\Psi_{X_{2}} \widetilde{\Pi} \Psi_{X_{3}}\right)$.

Proof. The proofs of (i)-(iv) are trivial, therefore omitted.

v) For all $\varepsilon_{j} \in \mathcal{E}$,

$$
\begin{aligned}
\beta_{X_{1} \widetilde{\Pi}\left(X_{2} \widetilde{\Pi} X_{3}\right)}\left(\varepsilon_{j}\right) & =\inf \left[\beta_{X_{1}}\left(\varepsilon_{j}\right), \beta_{X_{2} \widetilde{\Pi} X_{3}}\left(\varepsilon_{j}\right)\right] \\
& =\inf \left[\beta_{X_{1}}\left(\varepsilon_{j}\right), \inf \left[\beta_{X_{2}}\left(\varepsilon_{j}\right), \beta_{X_{3}}\left(\varepsilon_{j}\right)\right]\right] \\
& =\inf \left[\beta_{X_{1}}\left(\varepsilon_{j}\right),\left[\inf \left\{\underline{\beta_{X_{2}}}\left(\varepsilon_{j}\right), \underline{\beta_{X_{3}}}\left(\varepsilon_{j}\right)\right\}, \inf \left\{\overline{\beta_{X_{2}}}\left(\varepsilon_{j}\right), \overline{\beta_{X_{3}}}\left(\varepsilon_{j}\right)\right\}\right]\right] \\
& =\left[\inf \left\{\underline{\beta_{X_{1}}}\left(\varepsilon_{j}\right), \inf \left\{\underline{\beta_{X_{2}}}\left(\varepsilon_{j}\right), \underline{\beta_{X_{3}}}\left(\varepsilon_{j}\right)\right\}\right\}, \inf \left\{\overline{\beta_{X_{1}}}\left(\varepsilon_{j}\right), \inf \left\{\overline{\beta_{X_{2}}}\left(\varepsilon_{j}\right), \overline{\beta_{X_{3}}}\left(\varepsilon_{j}\right)\right\}\right\}\right] \\
& \left.=\inf \left\{\inf \left\{\underline{\beta_{X_{1}}}\left(\varepsilon_{j}\right), \underline{\beta_{X_{2}}}\left(\varepsilon_{j}\right)\right\}, \underline{\beta_{X_{3}}}\left(\varepsilon_{j}\right)\right\}, \inf \left\{\inf \left\{\overline{\beta_{X_{1}}}\left(\varepsilon_{j}\right), \overline{\beta_{X_{2}}}\left(\varepsilon_{j}\right)\right\}, \overline{\beta_{X_{3}}}\left(\varepsilon_{j}\right)\right\}\right] \\
& \left.=\inf \left[\inf \left\{\underline{\beta_{X_{1}}}\left(\varepsilon_{j}\right), \underline{\beta_{X_{2}}}\left(\varepsilon_{j}\right)\right\}, \inf \left\{\overline{\beta_{X_{1}}}\left(\varepsilon_{j}\right), \overline{\beta_{X_{2}}}\left(\varepsilon_{j}\right)\right\}\right], \beta_{X_{3}}\left(\varepsilon_{j}\right)\right] \\
& =\inf \left[\inf \left[\beta_{X_{1}}\left(\varepsilon_{j}\right), \beta_{X_{2}}\left(\varepsilon_{j}\right)\right], \beta_{X_{3}}\left(\varepsilon_{j}\right)\right] \\
& =\inf \left[\beta_{X_{1} \widetilde{\Pi} X_{2}}\left(\varepsilon_{j}\right), \beta_{X_{3}}\left(\varepsilon_{j}\right)\right]
\end{aligned}
$$




$$
\begin{aligned}
& =\beta_{\left(X_{1} \widetilde{\Pi} X_{2}\right) \widetilde{\Pi} X_{3}}\left(\varepsilon_{j}\right), \\
\psi_{X_{1} \widetilde{\Pi}\left(X_{2} \widetilde{\Pi} X_{3}\right)}\left(\varepsilon_{j}\right) & =\psi_{X_{1}}\left(\varepsilon_{j}\right) \cap \psi_{X_{2} \widetilde{\Pi} X_{3}}\left(\varepsilon_{j}\right) \\
& =\psi_{X_{1}}\left(\varepsilon_{j}\right) \cap\left(\psi_{X_{2}}\left(\varepsilon_{j}\right) \cap \psi_{X_{3}}\left(\varepsilon_{j}\right)\right) \\
& =\left(\psi_{X_{1}}\left(\varepsilon_{j}\right) \cap \psi_{X_{2}}\left(\varepsilon_{j}\right)\right) \cap \psi_{X_{3}}\left(\varepsilon_{j}\right) \\
& =\psi_{X_{1} \widetilde{\sqcap} X_{2}}\left(\varepsilon_{j}\right) \cap \psi_{X_{3}}\left(\varepsilon_{j}\right) \\
& =\psi_{\left(X_{1} \widetilde{\sqcap} X_{2}\right) \widetilde{\Pi} X_{3}}\left(\varepsilon_{j}\right) .
\end{aligned}
$$

Thus, we have $\Psi_{X_{1}} \widetilde{\Pi}\left(\Psi_{X_{2}} \widetilde{\Pi} \Psi_{X_{3}}\right)=\left(\Psi_{X_{1}} \widetilde{\Pi} \Psi_{X_{2}}\right) \widetilde{\Pi} \Psi_{X_{3}}$.

Proposition 3.16. Let $\Psi_{X_{1}}, \Psi_{X_{2}}, \Psi_{X_{3}} \in \operatorname{IVFPIFSS}(U)$. Then,

i) $\Psi_{X_{1}} \widetilde{\square}\left(\Psi_{X_{2}} \widetilde{\Pi} \Psi_{X_{3}}\right)=\left(\Psi_{X_{1}} \widetilde{\square} \Psi_{X_{2}}\right) \widetilde{\Pi}\left(\Psi_{X_{1}} \widetilde{\square} \Psi_{X_{3}}\right)$.

ii) $\Psi_{X_{1}} \widetilde{\Pi}\left(\Psi_{X_{2}} \widetilde{\sqcup} \Psi_{X_{3}}\right)=\left(\Psi_{X_{1}} \widetilde{\Pi} \Psi_{X_{2}}\right) \widetilde{\square}\left(\Psi_{X_{1}} \widetilde{\Pi} \Psi_{X_{3}}\right)$.

Proof. The proofs can be proved in a similar manner to the proofs of Propositions 3.12 and 3.15.

Proposition 3.17. Let $\Psi_{X_{1}}, \Psi_{X_{2}} \in \operatorname{IVFPIFSS}(U)$. Then, the following De-Morgan laws are valid.

i) $\left(\Psi_{X_{1}} \widetilde{\square} \Psi_{X_{2}}\right)^{c}=\Psi_{X_{1}}{ }^{c} \widetilde{\Pi} \Psi_{X_{2}}{ }^{c}$.

ii) $\left(\Psi_{X_{1}} \widetilde{\Pi} \Psi_{X_{2}}\right)^{c}=\Psi_{X_{1}}{ }^{c} \widetilde{\sqcup} \Psi_{X_{2}}{ }^{c}$.

Proof. i) For all $\varepsilon_{j} \in \mathcal{E}$,

$$
\begin{aligned}
& \beta_{\left(X_{1} \widetilde{\square} X_{2}\right)} \tilde{c}\left(\varepsilon_{j}\right)=1-\beta_{X_{1} \widetilde{\square} X_{2}}\left(\varepsilon_{j}\right) \\
& =1-\left[\sup \left(\underline{\beta_{X_{1}}}\left(\varepsilon_{j}\right), \underline{\beta_{X_{2}}}\left(\varepsilon_{j}\right)\right), \sup \left(\overline{\beta_{X_{1}}}\left(\varepsilon_{j}\right), \overline{\beta_{X_{2}}}\left(\varepsilon_{j}\right)\right)\right] \\
& =\left[1-\sup \left(\overline{\overline{\beta_{X_{1}}}}\left(\varepsilon_{j}\right), \overline{\overline{\beta_{X_{2}}}}\left(\varepsilon_{j}\right)\right), 1-\sup \left(\underline{\beta_{X_{1}}}\left(\varepsilon_{j}\right), \underline{\beta_{X_{2}}}\left(\varepsilon_{j}\right)\right)\right] \\
& =\left[\inf \left(1-\overline{\beta_{X_{1}}}\left(\varepsilon_{j}\right), 1-\overline{\beta_{X_{2}}}\left(\varepsilon_{j}\right)\right), \inf \left(1-\underline{\beta_{X_{1}}}\left(\varepsilon_{j}\right), 1-\underline{\beta_{X_{2}}}\left(\varepsilon_{j}\right)\right)\right] \\
& =\inf \left[\beta_{X_{1}} \tilde{c}\left(\varepsilon_{j}\right), \beta_{X_{2}} \tilde{c}\left(\varepsilon_{j}\right)\right] \\
& =\beta_{X_{1} \tilde{\tilde{n}} \tilde{X_{2}}} \tilde{c}\left(\varepsilon_{j}\right) \text {, } \\
& \psi_{\left(X_{1} \widetilde{\square} X_{2}\right) \tilde{c}}\left(\varepsilon_{j}\right)=\left(\psi_{X_{1} \widetilde{\square} X_{2}}\left(\varepsilon_{j}\right)\right)^{c} \\
& =\left(\psi_{X_{1}}\left(\varepsilon_{j}\right) \cup \psi_{X_{2}}\left(\varepsilon_{j}\right)\right)^{c} \\
& =\left(\psi_{X_{1}}\left(\varepsilon_{j}\right)\right)^{c} \cap\left(\psi_{X_{2}}\left(\varepsilon_{j}\right)\right)^{c} \\
& =\psi_{X_{1}} \tilde{c}\left(\varepsilon_{j}\right) \cap \psi_{X_{2}} \tilde{c}\left(\varepsilon_{j}\right) \\
& =\psi_{X_{1}} \tilde{\tilde{}} \widetilde{\Pi} X_{2} \tilde{c}\left(\varepsilon_{j}\right) \text {. }
\end{aligned}
$$

Hence, we obtain that $\left(\Psi_{X_{1}} \widetilde{\cup} \Psi_{X_{2}}\right)^{c}=\Psi_{X_{1}}{ }^{c} \widetilde{\Pi} \Psi_{X_{2}}{ }^{c}$.

The proof of (ii) can be proved similarly. 


\section{IVFPIFS-AGGREGATION OPERATORS}

In this section, the IVFPIFS-aggregation operators which create the aggregate interval-valued intuitionistic fuzzy sets from an IVFPIFS set and its interval-valued fuzzy parameter set are introduced. By using these operators, an algorithm for selection is proposed.

Definition 4.1. Let $\Psi_{X}=\left\{\left(\beta_{j} / \varepsilon_{j},\left\{\prec \gamma_{i j}^{+}, \gamma_{i j}^{-}>/ u_{i}: u_{i} \in U\right\}\right): \varepsilon_{j} \in \mathcal{E}\right\}$ be an IVFPIFS set, where $\gamma_{i j}=\prec$ $\gamma_{i j}^{+}, \gamma_{i j}^{-}>i=1,2, \ldots, n$ indicates an intuitionistic fuzzy value when the alternative $u_{i}$ is assessed with respect to the parameter $\varepsilon_{j}$ and $\beta_{j}=\left[\underline{\beta_{j}}, \overline{\beta_{j}}\right]$ indicates an interval-valued fuzzy value of the parameter $\varepsilon_{j}$. Then, the first IVFPIFS-aggregation operator, denoted by IVFPIFS $S_{a g g}^{1}$, is defined by

$$
\operatorname{IVFPIFS} S_{a g g}^{1}: \operatorname{IVFS}(\mathcal{E}) \times \operatorname{IVFPIFSS}(U) \rightarrow \operatorname{IVIFS}(U), \operatorname{IVFPIFS} S_{a g g}^{1}\left(X, \Psi_{X}\right)=\dot{\Psi}_{X}
$$

where $\dot{\Psi}_{X}=\left\{\left\langle\left[\underline{\dot{\gamma}_{i}^{+}}, \overline{\dot{\gamma}_{i}^{+}}\right],\left[\underline{\dot{\gamma}_{i}^{-}}, \overline{\dot{\gamma}_{i}^{-}}\right]>/ u_{i}: u_{i} \in U\right\}\right.$ which is an interval-valued intuitionistic fuzzy set on $U$.

Here, the membership degree $\dot{\gamma}_{i}^{+}=\left[\underline{\dot{\gamma}_{i}^{+}}, \overline{\dot{\gamma}_{i}^{+}}\right]$and the non-membership degree $\dot{\gamma}_{i}^{-}=\left[\underline{\dot{\gamma}_{i}^{-}}, \overline{\dot{\gamma}_{i}^{-}}\right]$of $u_{i} \in U$ is defined as follows:

$$
\dot{\gamma}_{i}^{+}=\left[1-\prod_{j=1}^{m}\left(1-\underline{\beta_{j}} \gamma_{i j}^{+}\right), 1-\prod_{j=1}^{m}\left(1-\overline{\beta_{j}} \gamma_{i j}^{+}\right)\right] \text {and } \dot{\gamma}_{i}^{-}=\left[\prod_{j=1}^{m} \underline{\beta_{j}} \gamma_{i j}^{-}, \prod_{j=1}^{m} \overline{\beta_{j}} \gamma_{i j}^{-}\right]
$$

where $m$ denotes the number of parameters in $\varepsilon$.

Definition 4.2. Let $\Psi_{X}=\left\{\left(\beta_{j} / \varepsilon_{j},\left\{\prec \gamma_{i j}^{+}, \gamma_{i j}^{-}>/ u_{i}: u_{i} \in U\right\}\right): \varepsilon_{j} \in \mathcal{E}\right\}$ be an IVFPIFS set, where $\gamma_{i j}=\prec$ $\gamma_{i j}^{+}, \gamma_{i j}^{-}>i=1,2, \ldots, n$ indicates an intuitionistic fuzzy value when the alternative $u_{i}$ is assessed with respect to the parameter $\varepsilon_{j}$ and $\beta_{j}=\left[\underline{\beta_{j}}, \overline{\beta_{j}}\right]$ indicates an interval-valued fuzzy value of the parameter $\varepsilon_{j}$. Then, the second IVFPIFS-aggregation operator, denoted by IVFPIFS 2 agg, is defined by

$$
\operatorname{IVFPIFS} S_{a g g}^{2}: \operatorname{IVFS}(\mathcal{E}) \times \operatorname{IVFPIFSS}(U) \rightarrow \operatorname{IVIFS}(U), \operatorname{IVFPIFS} S_{a g g}^{2}\left(X, \Psi_{X}\right)=\ddot{\Psi}_{X}
$$

where $\ddot{\Psi}_{X}=\left\{<\left[\underline{\ddot{\gamma}_{i}^{+}}, \bar{\gamma}_{i}^{+}\right],\left[\underline{\ddot{\gamma}_{i}^{-}}, \bar{\gamma}_{i}^{-}\right]>/ u_{i}: u_{i} \in U\right\}$ which is an interval-valued intuitionistic fuzzy set on $U$.

Here, the membership degree $\ddot{\gamma}_{i}^{+}=\left[\underline{\ddot{\gamma}_{i}^{+}}, \overline{\ddot{\gamma}_{i}^{+}}\right]$and the non-membership degree $\ddot{\gamma}_{i}^{-}=\left[\underline{\ddot{\gamma}_{i}^{-}}, \bar{\gamma}_{i}^{-}\right]$of $u_{i} \in U$ is defined as follows:

$$
\ddot{\gamma}_{i}^{+}=\left[\prod_{j=1}^{m} \underline{\beta_{j}} \gamma_{i j}^{+}, \prod_{j=1}^{m} \overline{\beta_{j}} \gamma_{i j}^{+}\right] \text {and } \ddot{\gamma}_{i}^{-}=\left[1-\prod_{j=1}^{m}\left(1-\underline{\beta_{j}} \gamma_{i j}^{-}\right), \prod_{j=1}^{m}\left(1-\overline{\beta_{j}} \gamma_{i j}^{-}\right)\right]
$$

where $m$ denotes the number of parameters in $\mathcal{E}$.

Now, we construct an algorithm using the interval-valued intuitionistic fuzzy sets which are found by utilizing IVFPIFS 1 agg and IVFPIFS 2 agg.

\section{Algorithm}

Step 1. Construct an IVFPIFS set $\Psi_{X}$ on $U$.

Step 2. By using the first IVFPIFS-aggregation operator given in Eq. (4.1), obtain the first aggregate interval-valued intuitionistic fuzzy set $\dot{\Psi}_{X}=\left\{\prec\left[\underline{\dot{\gamma}_{i}^{+}}, \overline{\dot{\gamma}_{i}^{+}}\right],\left[\underline{\dot{\gamma}_{i}^{-}}, \overline{\dot{\gamma}_{i}^{-}}\right]>/ u_{i}: u_{i} \in U\right\}$ of the IVFPIFS set $\Psi_{X}$ on $U$. 
Step 3. By using the second IVFPIFS-aggregation operator given in Eq. (4.2), obtain the second aggregate interval-valued intuitionistic fuzzy set $\ddot{\Psi}_{X}=\left\{<\left[\underline{\ddot{\gamma}_{i}^{+}}, \overline{\ddot{\gamma}_{i}^{+}}\right],\left[\underline{\ddot{\gamma}_{i}^{-}}, \overline{\ddot{\gamma}}_{i}^{-}\right]>/ u_{i}: u_{i} \in U\right\}$ of the IVFPIFS set $\Psi_{X}$ on $U$.

Step 4. Find the IVIF values $\prec \dddot{\gamma}_{i}^{+}, \dddot{\gamma}_{i}^{-}>=<\dot{\gamma}_{i}^{+}, \dot{\gamma}_{i}^{-}>\oplus \prec \ddot{\gamma}_{i}^{+}, \ddot{\gamma}_{i}^{-}>$for $i=1,2, \ldots, n$ (or $\prec \dddot{\gamma}_{i}^{+}, \dddot{\gamma}_{i}^{-}>=<\dot{\gamma}_{i}^{+}, \dot{\gamma}_{i}^{-}>\otimes \prec \ddot{\gamma}_{i}^{+}, \ddot{\gamma}_{i}^{-}>$) by employing any of the operations $\oplus$ and $\otimes$ given in Definition 2.7.

Step 5. Compare the IVIF values by using the score function and accuracy function of $\dddot{\gamma}_{i}=\prec \dddot{\gamma}_{i}^{+}, \dddot{\gamma}_{i}^{-}>$ for $i=1,2, \ldots, n$.

Step 6. As a result of these comparisons, obtain opportune element of $U$ which is denoted and defined by

$$
U_{\text {opp }}=\left\{u_{i}: u_{i} \in U \text { and } \dddot{\gamma}_{i}>\dddot{\gamma}_{i^{\prime}} \text { for all } i^{\prime} \neq i\right\}
$$

Let us give two illustrative examples to better understand the steps of this algorithm.

Example 4.3. Let's consider a retailer planning to open a new store in the city. There are five streets to be selected, i.e. $U=\left\{\mathrm{u}_{1}, \mathrm{u}_{2}, \mathrm{u}_{3}, \mathrm{u}_{4}, \mathrm{u}_{5}\right\}$. Three parameters are considered: $\varepsilon_{1}=$ traffic, $\varepsilon_{2}=$ rent price, $\varepsilon_{3}=$ crowdedness and $\varepsilon_{4}=$ competition. We aim to determine opportune street, where the retailer can open a new store. For this purpose, we apply the above algorithm as follows:

Step 1. After evaluating the streets under the specified parameters, the IVFPIFS set is generated as below:

$\Psi_{X}=\left\{\left([0.4,0.7] / \varepsilon_{1}\left\{<0.6,0.3>/ u_{1},<0.5,0.3>/ u_{2},<0.4,0.6>/ u_{3},<0.4,0.3>/ u_{4}\right.\right.\right.$,

$\left.\left.\prec 0.7,0.1>/ u_{5}\right\}\right),\left([0.6,0.9] / \varepsilon_{2},\left\{<0.4,0.5>/ u_{1},<0.7,0.1>/ u_{2},<0.2,0.7>/ u_{3}\right.\right.$,

$\left.\left.\prec 0.35,0.35>/ u_{4},<0.5,0.3>/ u_{5}\right\}\right),\left([0.5,0.8] / \varepsilon_{3}\left\{<0.7,0.2>/ u_{1},<0.85,0.1>/ u_{2}\right.\right.$

$\left.\left.\prec 0.1,0.7>/ u_{3},<0.4,0.6>/ u_{4},<0.6,0.4>/ u_{5}\right\}\right),\left([0.3,0.6] / \varepsilon_{4},\left\{<0.5,0.5>/ u_{1}\right.\right.$,

$\left.\left.\left.\prec 0.6,0.4>/ u_{2}, \prec 0.25,0.7>/ u_{3}, \prec 0.2,0.4>/ u_{4}, \prec 0.85,0.1>/ u_{5}\right\}\right)\right\}$.

In order to follow the steps of the algorithm more easily, let's present this IVFPIFS set $\Psi_{X}$ with the table as follows.

Table 1: The tabular form of IVFPIFS set $\Psi_{\mathrm{X}}$.

\begin{tabular}{|c|c|c|c|c|}
\hline$\Psi_{X}$ & {$[0.4,0.7] / \varepsilon_{1}$} & {$[0.6,0.9] / \varepsilon_{2}$} & {$[0.5,0.8] / \varepsilon_{3}$} & {$[0.3,0.6] / \varepsilon_{4}$} \\
\hline$u_{1}$ & $\prec 0.6,0.3 \succ$ & $\prec 0.4,0.5>$ & $\prec 0.7,0.2 \succ$ & $\prec 0.5,0.5>$ \\
\hline$u_{2}$ & $\prec 0.5,0.3 \succ$ & $\prec 0.7,0.1>$ & $\prec 0.85,0.1 \succ$ & $\prec 0.6,0.4 \succ$ \\
\hline$u_{3}$ & $\prec 0.4,0.6 \succ$ & $\prec 0.2,0.7>$ & $\prec 0.1,0.7 \succ$ & $\prec 0.25,0.7 \succ$ \\
\hline$u_{4}$ & $\prec 0.4,0.3 \succ$ & $\prec 0.35,0.35 \succ$ & $\prec 0.4,0.6 \succ$ & $\prec 0.2,0.4 \succ$ \\
\hline$u_{5}$ & $\prec 0.7,0.1 \succ$ & $\prec 0.5,0.3 \succ$ & $\prec 0.6,0.4 \succ$ & $\prec 0.85,0.1 \succ$ \\
\hline
\end{tabular}

The value in the $i$ th row and jth column of this table denotes the intuitionistic fuzzy value of the alternative $u_{i}$ evaluated according to the parameter $\varepsilon_{j}$.

Step 2. The first aggregate interval-valued intuitionistic fuzzy set $\dot{\Psi}_{X}$ of the IVFPIFS set $\Psi_{X}$ is obtained as in Table 2. 
Table 2: The tabular form of $\dot{\Psi}_{X}$.

\begin{tabular}{|c|c|}
\hline$\dot{\gamma}_{i} / u_{\mathrm{i}}$ & $\dot{\gamma}_{i}=\prec \dot{\gamma}_{i}^{+}, \dot{\gamma}_{i}^{-}>=<\left[\dot{\gamma}_{i}^{+}, \overline{\dot{\gamma}_{i}^{+}}\right],\left[\dot{\gamma}_{i}^{-}, \overline{\dot{\gamma}_{i}^{-}}\right]>$ \\
\hline$u_{1}$ & $\prec[0.680876,0.8856704],[0.00054,0.004536] \succ$ \\
\hline$u_{2}$ & $\prec[0.781224,0.9507456],[0.0000432,0.00036288] \succ$ \\
\hline$u_{3}$ & $\prec[0.350428,0.5383072],[0.0074088,0.06223392] \succ$ \\
\hline$u_{4}$ & $\langle[0.5009728,0.70486912],[0.0009072,0.00762048]>$ \\
\hline$u_{5}$ & $\prec[0.737164,0.9285286],[0.0000432,0.00036288] \succ$ \\
\hline
\end{tabular}

Step 3. The second aggregate interval-valued intuitionistic fuzzy set $\ddot{\Psi}_{X}$ of the IVFPIFS set $\Psi_{X}$ is obtained as in Table 3.

Table 3: The tabular form of $\ddot{\Psi}_{X}$.

\begin{tabular}{|c|c|}
\hline$\ddot{\gamma}_{i} / u_{\mathrm{i}}$ & $\ddot{\gamma}_{i}=<\ddot{\gamma}_{i}^{+}, \ddot{\gamma}_{i}^{-}>=<\left[\underline{\ddot{\gamma}_{i}^{+}}, \overline{\bar{\gamma}_{i}^{+}}\right],\left[\underline{\ddot{\gamma}_{i}^{-}}, \overline{\ddot{\gamma}_{i}^{-}}\right]>$ \\
\hline$u_{1}$ & $\prec[0.003024,0.0254016],[0.52876,0.744514] \succ$ \\
\hline$u_{2}$ & $\prec[0.006426,0.0539784],[0.3084608,0.49734512] \succ$ \\
\hline$u_{3}$ & $\prec[0.000072,0.0006048],[0.7736492,0.94523408] \succ$ \\
\hline$u_{4}$ & $\prec[0.0004032,0.00338688],[0.5717568,0.78613752] \succ$ \\
\hline$u_{5}$ & $\prec[0.006426,0.0539784],[0.3891328,0.56604712] \succ$ \\
\hline
\end{tabular}

Step 4. By using the operation $\oplus$ in Definition 2.7, we have Table 4.

Table 4: The tabular form of $\dddot{\gamma}_{i}$ obtained by using $\oplus$.

\begin{tabular}{|c|l|}
\hline$\dddot{\gamma}_{i} / u_{\mathrm{i}}$ & $\dddot{\gamma}_{i}=\prec \dddot{\gamma}_{i}^{+}, \dddot{\gamma}_{i}^{-}>=\prec \dot{\gamma}_{i}^{+}, \dot{\gamma}_{i}^{-}>\oplus \prec \ddot{\gamma}_{i}^{+}, \ddot{\gamma}_{i}^{-}>$ \\
\hline$u_{1}$ & $\prec[0.68184103,0.8887455],[0.0002855304,0.003377116] \succ$ \\
\hline$u_{2}$ & $\prec[0.78262985,0.95340427],[0.0000133255,0.0001804766] \succ$ \\
\hline$u_{3}$ & $\prec[0.35047475,0.53858643],[0.005731812,0.05882562] \succ$ \\
\hline$u_{4}$ & $\prec[0.50114701,0.70586912],[0.0005186978,0.005990745] \succ$ \\
\hline$u_{5}$ & $\prec[0.73885298,0.93251502],[0.00001681053,0.0002054072] \succ$ \\
\hline
\end{tabular}

Step 5. We give the results of score function of $\dddot{\gamma}_{i}=<\dddot{\gamma}_{i}^{+}, \dddot{\gamma}_{i}^{-}>$for $i=1,2,3,4,5$ as in Table 5 .

Table 5: The tabular form of the results of score function for $\oplus$.

\begin{tabular}{|c|c|c|c|c|c|}
\hline $\mathrm{i} / \operatorname{Scr}\left(\dddot{\gamma}_{i}\right)$ & 1 & 2 & 3 & 4 & 5 \\
\hline $\operatorname{Scr}\left(\dddot{\gamma}_{i}\right)$ & 0.78346194 & 0.86792016 & 0.41225188 & 0.60025334 & 0.83557289 \\
\hline
\end{tabular}

By Definition 2.6, we have $\dddot{\gamma}_{2}>\dddot{\gamma}_{5}>\dddot{\gamma}_{1}>\dddot{\gamma}_{4}>\dddot{\gamma}_{3}$ since $\operatorname{Scr}\left(\dddot{\gamma}_{2}\right)>\operatorname{Scr}\left(\dddot{\gamma}_{5}\right)>\operatorname{Scr}\left(\dddot{\gamma}_{1}\right)>\operatorname{Scr}\left(\dddot{\gamma}_{4}\right)>$ $\operatorname{Scr}\left(\dddot{\gamma}_{3}\right)$.

Here, if the results obtained by using the score function for some IVIF values are the same, then new comparison results will be obtained by using the accuracy function for these IVIF values. 
Step 6. As a result of these comparisons, we obtain the opportune element of $U$ is $U_{\text {opp }}=\left\{u_{2}\right\}$. Thus, we can say that the most appropriate street in the city for a new store is $u_{2}$.

Example 4.4. Let us consider Example 4.3. If the operation $\otimes$ is taken instead of the operation $\oplus$ in Step 4 , then the following table is obtained.

Table 6: The tabular form of $\dddot{\gamma}_{i}$ obtained by using $\otimes$.

\begin{tabular}{|c|l|}
\hline$\dddot{\gamma}_{i} / u_{i}$ & $\dddot{\gamma}_{i}=\prec \dddot{\gamma}_{i}^{+}, \dddot{\gamma}_{i}^{-}>=\prec \dot{\gamma}_{i}^{+}, \dot{\gamma}_{i}^{-}>\otimes \prec \ddot{\gamma}_{i}^{+}, \ddot{\gamma}_{i}^{-}>$ \\
\hline$u_{1}$ & $\prec[0.002058969,0.02249745],[0.52901447,0.74567288]>$ \\
\hline$u_{2}$ & $\prec[0.005020145,0.05131973],[0.30849067,0.49752752] \succ$ \\
\hline$u_{3}$ & $\prec[0.0000252308,0.0003255682],[0.77532619,0.94864238] \succ$ \\
\hline$u_{4}$ & $\prec[0.0002019922,0.002387307],[0.5721453,0.78776725]>$ \\
\hline$u_{5}$ & $\prec[0.004737016,0.05012049],[0.38915919,0.56947051]>$ \\
\hline
\end{tabular}

Thus, we have the results of score function of $\dddot{\gamma}_{i}=\prec \dddot{\gamma}_{i}^{+}, \dddot{\gamma}_{i}^{-}>$for $i=1,2,3,4,5$ as in Table 7 .

Table 7: The tabular form of the results of score function for $\otimes$.

\begin{tabular}{|c|c|c|c|c|c|}
\hline $\mathrm{i} / \operatorname{Scr}\left(\dddot{\gamma}_{i}\right)$ & 1 & 2 & 3 & 4 & 5 \\
\hline $\operatorname{Scr}\left(\dddot{\gamma}_{i}\right)$ & -0.6250654 & -0.3748392 & -0.8618089 & -0.6786616 & -0.4518861 \\
\hline
\end{tabular}

Then, we obtain $\dddot{\gamma}_{2}>\dddot{\gamma}_{5}>\dddot{\gamma}_{1}>\dddot{\gamma}_{4}>\dddot{\gamma}_{3}$ since $\operatorname{Scr}\left(\dddot{\gamma}_{2}\right)>\operatorname{Scr}\left(\dddot{\gamma}_{5}\right)>\operatorname{Scr}\left(\dddot{\gamma}_{1}\right)>\operatorname{Scr}\left(\dddot{\gamma}_{4}\right)>\operatorname{Scr}\left(\dddot{\gamma}_{3}\right)$. Hence, according to these comparisons, we say that the opportune element of $U$ is $U_{o p p}=\left\{u_{2}\right\}$.

By Tables 6 and 7, it is easily seen that the values $\dddot{\gamma}_{i}$ and $\operatorname{Scr}\left(\dddot{\gamma}_{i}\right)$ for each $u_{i}(i=1,2,3,4,5)$ have changed. However, the comparison results and also the opportune element are the same.

\section{CONCLUSION}

In this paper, we defined the interval-valued fuzzy parameterized intuitionistic fuzzy soft sets by combining the interval-valued fuzzy sets and the intuitionistic fuzzy soft sets from parameterization point of view. Their basic operations and some interesting results were introduced. Also, we proposed a decision making model based on the IVFPIFS-aggregation operators, which is described in this study.

We think that the IVFPIFS sets can be applied not only to decision making problems but also problems of many fields containing uncertainty. Moreover, we hope that the findings in this work will help researchers promote and enhance the further study on intuitionistic fuzzy soft sets.

\section{REFERENCES}

[1] Zadeh, L.A., Fuzzy sets, Inform. Control, 8 (1965) 338-353.

[2] Sambuc, R., Fonctions $\Phi$-floues. Application a l'aide au diagnostic en pathologie thyroidienne. $\mathrm{Ph}$. D. Thesis, Univ. Marseille, France, 1975.

[3] Atanassov, K.T., Intuitionistic fuzzy sets, Fuzzy Set. Syst., 20 (1986) 87-96.

[4] Atanassov, K. and Gargov, G., Interval-valued intuitionistic fuzzy sets, Fuzzy Set. Syst., 31 (1989) 343-349.

[5] Molodtsov, D., Soft set theory-first results, Comput. Math. Appl., 37 (1999) 19-31. 
[6] Ali, M.I., Feng, F., Liu, X., Min, W.K. and Shabir, M., On some new operations in soft set theory, Comput. Math. Appl., 57 (2009) 1547-1553.

[7] Maji, P.K., Biswas, R. and Roy, A.R., Soft set theory, Comput. Math. Appl., 45 (2003) 555-562.

[8] Sezgin, A. and Atagün, A.O., On operations of soft sets, Comput. Math. Appl., 61 (2011) 14571467.

[9] Aktaş, H. and Çağman, N., Soft sets and soft groups, Inform. Sci., 177 (2007) 2726-2735.

[10] Feng, F., Jun, Y.B. and Zhao, X., Soft semirings, Comput. Math. Appl., 56 (2008) 2621-2628.

[11] Acar, U., Koyuncu, F. and Tanay, B., Soft sets and soft rings, Comput. Math. Appl., 59 (2010) 3458-3463.

[12] Atagün, A.O. and Sezgin, A., Soft substructures of rings, fields and modules, Comput. Math. Appl., 61 (2011) 592-601.

[13] Sezgin, A., Atagün, A.O. and Aygün, E., A note on soft near-rings and idealistic soft near-rings, Filomat, 25 (2011) 53-68.

[14] Çağman, N., Çıtak, F. and Enginoğlu, S., FP-soft set theory and its applications, Ann. Fuzzy Math. Inform., 2 (2011) 219-226.

[15] Zorlutuna, I. and Atmaca, S., Notes on fuzzy parametrized soft sets, Cumhuriyet Science Journal, 39 (2018) 818-827.

[16] Çağman, N. and Deli, I., Products of FP-soft sets and their applications, Hacet. J. Math. Stat., 41 (2012) 365-374.

[17] Çağman, N. and Deli, I., Means of FP-soft sets and their applications, Hacet. J. Math. Stat., 41 (2012) 615-625.

[18] Deli, I. and Çağman, N., Relations on FP-soft sets applied to decision making problems, Journal of New Theory, 3 (2015) 98-107.

[19] Deli, I. and Çağman, N., Intuitionistic fuzzy parameterized soft set theory and its decision making, Appl. Soft Comput., 28 (2015) 109-113.

[20] Deli, I. and Karataş, S., Interval valued intuitionistic fuzzy parameterized soft set theory and its decision making, J. Intell. Fuzzy Syst., 30 (2016) 2073-2082.

[21] Çağman, N., Enginoğlu, S. and Çıtak, F., Fuzzy soft set theory and its applications, Iran. J. Fuzzy Syst., 8 (2011) 137-147.

[22] Atagün, A.O., Kamac1, H. and Oktay, O., Reduced soft matrices and generalized products with applications in decision making, Neural Comput. Applic., 29 (2018) 445-456.

[23] Çağman, N. and Enginoğlu, S., Soft matrix theory and its decision making, Comput. Math. Appl., 59 (2010) 3308-3314.

[24] Çağman, N. and Enginoğlu, S., Fuzzy soft matrix theory and its application in decision making, Iran. J. Fuzzy Syst., 9 (2012) 109-119.

[25] Kamacı, H., Atagün, A.O. and Sönmezoğlu, A., Row-products of soft matrices with applications in multiple-disjoint decision making, Appl. Soft Comput., 62 (2018) 892-914.

[26] Kamac1, H., Atagün, A.O. and Aygün, E., Difference operations of soft matrices with applications in decision making, Punjab Univ. J. Math., 51 (2019) 1-21.

[27] Petroudi, S.H.J., Nabati, Z. and Yaghobi, A., Some new results on fuzzy soft matrices, Turkish Journal of Fuzzy Systems, 8 (2017) 52-62.

[28] Maji, P.K., Biswas, R. and Roy, A.R., Intuitionistic fuzzy soft sets, The Journal of Fuzzy Mathematics, 9 (2001) 677-692.

[29] Xu, Y.-J., Sun, Y.-K. and Li, D.-F., Intuitionistic fuzzy soft set, $2^{\text {nd }}$ International Workshop on Intelligent Systems and Applications IEEE, Wuhan, China, 2010.

[30] Çağman, N. and Karataş, S., Intuitionistic fuzzy soft set theory and its decision making, J. Intell. Fuzzy Syst., 24 (2013) 829-836. 
[31] Deli, I. and Çağman, N., Similarity measure of IFS-sets and its application in medical diagnosis, Ann. Fuzzy Math. Inform., 11 (2016) 841-854.

[32] Broumi, S., Majumdar, P. and Smarandache, F., New operations on intuitionistic fuzzy soft sets based on first Zadeh's logical operators, Journal of New Results in Science, 4 (2014) 71-81.

[33] Chetia, B., Das, P.K., Some results of intuitionistic fuzzy soft sets and its application in decision making, Appl. Math. Sci., 7 (2013) 4693-4712.

[34] Deli, I., npn-soft sets theory and applications, Ann. Fuzzy Math. Inform., 10 (2015) 847-862.

[35] Deli, I., Interval-valued neutrosophic soft sets and its decision making, Int. J. Mach. Learn. Cyber., 8 (2017) 665-676.

[36] Deli, I., Eraslan, S. and Çağman, N., ivnpiv-neutrosophic soft sets and their decision making based on similarity measure, Neural Comput. Applic., 29 (2018) 187-203.

[37] Dinda, B. and Samanta, T.K., Relations on intuitionistic fuzzy soft sets, General Mathematics Notes., 1 (2010) 74-83.

[38] Karaaslan, F., Intuitionistic fuzzy parameterized intuitionistic fuzzy soft sets with applications in decision making, Ann. Fuzzy Math. Inform., 11 (2016) 607-619.

[39] Yin, Y., Li, H. and Jun, Y.B., On algebraic structure of intuitionistic fuzzy soft sets, Comput. Math. Appl., 64 (2012) 2896-2911.

[40] Tan, C., A multi-criteria interval-valued intuitionistic fuzzy group decision making with Choquet integral-based TOPSIS, Expert Syst. Appl., 38 (2011) 3023-3033.

[41] Xu, Z., Choquet integrals of weighted intuitionistic fuzzy information, Inf. Sci., 180 (2010) 726736.

[42] Çağman, N. and Enginoğlu, S., Soft set theory and uni-int decision making, Eur. J. Oper. Res., 207 (2010) 615-625. 